\title{
MEASURE-THEORETIC QUANTIFIERS AND HAAR MEASURE
}

\author{
RUSSELL LYONS
}

\begin{abstract}
Measure-theoretic quantifiers are introduced as convenient notation and to facilitate certain applications of Fubini's theorem. They are used to prove the uniqueness of Haar measure and to give some conditions involving translation which imply absolute continuity of another measure.
\end{abstract}

It is a remarkable fact that on a topological group with Haar measure, every measure is almost absolutely continuous with respect to Haar measure in a sense given below. (The case of Lebesgue measure occurs in [3].) The proof, which is a simple application of Fubini's theorem, can be expressed compactly using measuretheoretic quantifiers. As corollaries of this fact, we may deduce the uniqueness of Haar measure and some conditions involving translation which imply absolute continuity of another measure. (Again, compare [3].) The author wishes to acknowledge valuable conversations with Allen Shields which led to these results.

Since this paper essentially consists of applications of Fubini's theorem, we will make the standing hypothesis that all measures are $\sigma$-finite, possibly complexvalued.

If $P(t)$ is a predicate, let us write $\forall^{e} t[\mu] P(t)$ for "for almost every $t[\mu] P(t)$ " and $\exists^{m} t[\mu] P(t)$ for "there exist many $t[\mu] P(t)$ ", i.e., "there is a set of positive $\mu$-measure such that for $t$ in this set we have $P(t)$ ". These measure-theoretic quantifiers enjoy the "interchange property" that ordinary quantifiers have when the predicate is measurable in the following sense.

DEFINITION. If $X$ is a measurable space and $P(x), x \in X$, is a predicate, then $P$ is called measurable if the set $\{x: P(x)\}$ is measurable.

For example, if $E$ is a Borel subset of a topological group $G$ and $P(s, t)$ is the predicate "st $\notin E$ " on $G \times G$, or " $\chi_{E}(s t)=0$ ", where $\chi_{E}$ is the characteristic function of $E$, then $P$ is Borel-measurable.

The interchange property we mentioned is as follows.

Proposition 1. Let $(X, \mu)$ and $(Y, \nu)$ be positive measure spaces and $P(x, y)$ be a measurable predicate on $(X \times Y, \mu \times \nu)$. Then

$$
\forall^{e} x[\mu] \forall^{e} y[\nu] P(x, y) \text { if and only if } \forall^{e} y[\nu] \forall^{e}[\mu] P(x, y) \text {. }
$$

Proof. Let $E=\{(x, y): \neg P(x, y)\}, E_{(x)}=\{y: \neg P(x, y)\}$ and $E^{(y)}=$ $\{x: \neg P(x, y)\}$, where $\neg$ means "not". If $\forall^{e} x[\mu] \forall^{e} y[\nu] P(x, y)$, then $\forall^{e} x[\mu] \nu E_{(x)}=$

Received by the editors October 21, 1981.

1980 Mathematics Subject Classification. Primary 43A05.

Key words and phrases. Quantifiers, Fubini's theorem, Haar measure, translation, absolute continuity. 
0, whence by Fubini's theorem,

$$
\begin{aligned}
0 & =\int \nu E_{(x)} d \mu(x)=\iint \chi_{E}(x, y) d \nu(y) d \mu(x) \\
& =\iint \chi_{E}(x, y) d \mu(x) d \nu(y)=\int \mu E^{(y)} d \nu(y) .
\end{aligned}
$$

Since $\mu, \nu \geq 0$, it follows that $\forall^{e} y[\nu] \mu E^{(y)}=0$.

We may also interchange the $\exists^{m}$ quantifiers under the same hypotheses, as is easily seen from the fact that

$$
\neg \forall^{e} x P(x) \text { if and only if } \exists^{m} x \neg P(x) .
$$

It is now a simple matter to prove that every measure is almost absolutely continuous.

THEOREM 2. Let $G$ be a topological group, $\mu$ a positive left-invariant Borel measure (i.e., a left Haar measure), $\nu$ any Borel measure, and $E$ any set of $\mu$-measure 0. Then $\forall^{e} t[\mu] \nu\left(E t^{-1}\right)=0$.

Thus, while $E$ itself may not have $\nu$-measure 0 , almost every translate of $E$ does. For the proof, we will use the example above of a measurable predicate. Note that $\sigma$-finiteness is necessary, as the counting measure shows.

ProOF. We have $\forall s \mu\left(s^{-1} E\right)=0$, or $\forall s \forall^{e} t[\mu]$ st $\notin E$. Hence $\forall^{e} t[\mu] \forall^{e} s[|\nu|] s t$ $\notin E$, where $|\nu|$ is the absolute value of $\nu$.

If it happens that $\nu$ is a right Haar measure, then since $\nu\left(E t^{-1}\right)=0$ for some $t$, it follows that $\nu E=0$. Hence

COROLLARY 3. If $\mu$ and $\nu$ are left and right Haar measures, respectively, on a topological group, then $\nu \ll \mu \ll \nu$.

Note that if $\check{\mu}(E) \equiv \mu\left(E^{-1}\right)$, then $\check{\mu}$ is right Haar if $\mu$ is left Haar and vice versa. Since $\mu \ll \check{\mu} \ll \mu$, Theorem 2 holds in the case where $\mu$ is right Haar. Also, as the proof shows, it holds if $\mu$ is translation-invariant only on the sets of measure 0 . Clearly, the converse holds as well.

To show that Haar measure is unique (up to a multiplicative constant), we require the following lemma. For a function $f(s)$, let $f_{t}(s)=f\left(s t^{-1}\right)$.

LEMMA 4. Let $G$ be a topological group with left or right Haar measure $\mu$ and another positive Borel measure $\nu$. Suppose that $f$ is a measurable function such that $\forall^{e} t[\mu] f=f_{t}$ a.e. $[\nu]$. Then $f$ is equal to a constant a.e. $[\nu]$.

ProOF. Since $\forall^{e} t[\mu] \forall^{e} s[\nu] f(s)=f_{t}(s)$, we have $\forall^{e} s[\nu] \forall^{e} t[\mu] f(s)=f\left(s t^{-1}\right)$. For any such $s$, let $E=\left\{t: f(s) \neq f\left(s t^{-1}\right)\right\}$. Thus $\mu E=0$. If $\mu$ is left Haar, then since $\mu\left(E^{-1}\right)=\breve{\mu}(E)=0$ by Corollary $3, \mu\left(s E^{-1}\right)=0$; if $\mu$ is right Haar, then $\mu\left(E s^{-1}\right)=0$, whence by Corollary 3, $\mu\left(s E^{-1}\right)=\check{\mu}\left(E s^{-1}\right)=0$. Thus $\mu\left(s E^{-1}\right)=0$ in both cases, or $\forall^{e} t[\mu] f(s)=f(t)$. That is, $f$ is a constant a.e. $[\mu]$. Since this constant is $f(s)$ for almost any $s[\nu]$, it follows that $f$ is constant a.e. $[\nu]$.

We remark that if $\mu=\nu=$ Lebesgue measure, then a simple argument using points of density shows that it suffices to have $f=f_{t}$ a.e. for a dense set of $t$. On the other hand, it does not in general suffice to assume that $\forall^{e} t[\nu] f=f_{t}$ a.e. $[\nu]$, as the following example will demonstrate: Let $G=[-\pi, \pi)$ be the circle and 
$\nu$ have support in $E=\{0\} \cup\left[\frac{2}{3}, 1\right]$, where $\nu(\{0\})=1$ and $\nu \mid\left[\frac{2}{3}, 1\right]$ is Lebesgue measure; let $f=\chi-E$.

We shall only need the lemma in the case where $\mu=\nu$ is left Haar. We remind the reader that for our proof, $\sigma$-finiteness is assumed, though not regularity.

THEOREM 5. If $\mu$ and $\nu$ are left Haar measures on a topological group $G$, then there is a constant $c$ such that $\nu=c \mu$.

Proof. By Corollary $3, \nu \ll \check{\mu} \ll \mu$, or $\nu \ll \mu$. Let $f=[d \nu / d \mu]$ be the Radon-Nikodym derivative. Then for all $t$ we have $f_{t}=\left[d \nu_{t} / d \mu_{t}\right]=[d \nu / d \mu]=f$ a.e. $[\mu]$, where $\nu_{t}(E)=\nu\left(E t^{-1}\right)$. Thus the result follows from the lemma.

Theorem 2 may be used to characterize absolute continuity as follows. (In [2], the result is proved under the hypothesis of commutativity.)

PROPOSITION 6. Let $G$ be a topological group with a regular left or right Haar measure $\mu$. Let $\nu$ be any Borel measure on $G$. Then $\nu \ll \mu$ if and only if $\nu\left(E t^{-1}\right)$ is continuous at the identity as a function of $t$ for every set $E$ of $\mu$-measure 0 .

ProOF. Suppose $\nu \ll \mu$ and $\mu E=0$. An argument like that in the proof of Lemma 4 shows that $\mu\left(E t^{-1}\right)=0$, whence $\nu\left(E t^{-1}\right)=0$ for all $t$.

Conversely, if $\mu E=0$ and $\nu\left(E t^{-1}\right)$ is continuous at the identity, then by Theorem $2, \nu\left(E t^{-1}\right)=0$ a.e. $[\mu]$. Since open sets have positive $\mu$-measure by regularity of $\mu$, continuity shows that $\nu\left(E t^{-1}\right)=0$ at the identity: $\nu E=0$.

If we consider the space $L^{1}(\nu)$, then we find that translation behaves nicely in even the most rudimentary ways only if $\nu$ is absolutely continuous. First we prove

LEMMA 7. If $\mu, \nu$ are finite left and right Haar measures, respectively, on a topological group $G$, then $(\mu G) \nu=(\nu G) \mu$.

Proof. For any Borel set $E$,

$$
\begin{aligned}
(\mu G)(\nu E) & =\int \nu\left(E t^{-1}\right) d \mu(t)=\iint \chi_{E}(s t) d \nu(s) d \mu(t) \\
& =\int \mu\left(s^{-1} E\right) d \nu(s)=(\nu G)(\mu E)
\end{aligned}
$$

by Fubini's theorem.

PROPOSITION 8. Let $\mu$ be a left Haar measure on a topological group $G$ and $\nu$ any positive Borel measure. If $f_{t} \in L^{1}(\nu)$ for every $f \in L^{1}(\nu)$ and every $t \in G$, then $\nu \ll \mu$. If $\nu G<\infty$, then $[d \nu / d \mu] \in L^{\infty}(\mu)$. If $\mu G<\infty$, then $[d \nu / d \mu]^{-1} \in L^{\infty}(\mu)$.

Proof. Let $\mu E=0$. By Theorem 2, there is a $t$ such that $\nu\left(E t^{-1}\right)=0$. Let $f=\infty \cdot \chi_{E t^{-1}}$. Then $f=0$ a.e. $[\nu]$, whence $f \in L^{1}(\nu)$ and so $f_{t} \in L^{1}(\nu)$. But $f_{t}=\infty \cdot \chi_{E}$, whence $\nu E=0$.

Let $g=[d \nu / d \mu]$. If $g \notin L^{\infty}(\mu)$, then the closed graph theorem, for example, shows that there is a nonnegative $f \in L^{1}(\mu)$ such that $f g \notin L^{1}(\mu)$. Thus $f \notin L^{1}(\nu)$, whence $f_{t^{-1}} \notin L^{1}(\nu)$ and Fubini's theorem gives, if $\nu G<\infty$,

$$
\begin{aligned}
\infty & =\int\left(\int f_{t^{-1}}(s) d \nu(s)\right) d \mu(t)=\iint f(s t) g(s) d \mu(s) d \mu(t) \\
& =\iint f(s t) g(s) d \mu(t) d \mu(s)=\int\|f\|_{L^{1}(\mu)} g(s) d \mu(s) \\
& =\|f\|_{L^{1}(\mu)} \cdot\|g\|_{L^{1}(\mu)}<\infty,
\end{aligned}
$$

a contradiction. 
Now if $\mu G<\infty$, let $f \in L^{1}(\nu)$. Since $\forall t f_{t} \in L^{1}(\nu)$, we have

$$
\begin{aligned}
\infty & >\int\left|f_{t^{-1}}(s)\right| d \nu(s)=\int|f(s t)| g(s) d \mu(s) \\
& =\int|f(u)| g\left(u t^{-1}\right) d \mu(u)=\int g\left(t u^{-1}\right)|f(u)| d \mu(u) \\
& =(g *|f|)(t),
\end{aligned}
$$

where $\gamma(t)=g\left(t^{-1}\right)$, using the fact that $\mu=\check{\mu}$ is right Haar by Lemma 7. By [1], this implies that $f \in L^{1}(\mu)$. That is, $L^{1}(\nu) \subseteq L^{1}(\mu)$. The closed graph theorem shows that for some $M,\|f\|_{L^{1}(\mu)} \leq M \cdot\|f\|_{L^{1}(\nu)}$ for all $f \in L^{1}(\nu)$. Letting $f=\chi_{E}$, we find that $\mu E \leq M \int_{E} g d \mu$, whence $g \geq 1 / M$ a.e. $[\mu]$. That is, $g^{-1} \in L^{\infty}(\mu)$.

The necessity of the finiteness conditions may be seen by easy examples on the real line.

It is also easy to deduce from Theorem 2 that if $\nu$ has the property that $f=g$ a.e. $[\nu]$ implies $f_{t}=g_{t}$ a.e. $[\nu]$ for all $t \in G$, then again $\nu \ll \mu$.

\section{REFERENCES}

1. D. A. Lind, Convolutions and absolute continuity, Proc. Amer. Math. Soc. 39 (1973), 347-348.

2. Walter Rudin, Measure algebras on abelian groups, Bull. Amer. Math. Soc. 65 (1959), $227-247$.

3. Stanislaw Saks, Theory of the integral, 2nd rev. ed., English transl., Stechert, New York, 1937, pp. 91-92.

Department of MAThematics, University of Michigan, ANN ARBor, MichiGAN 48109 